\title{
Artificial neural network based gynaecological image-guided adaptive brachytherapy treatment planning correction of intra-fractional organs at risk dose variation
}

\author{
Ramin Jaberi, PhD', Zahra Siavashpour, PhD², Prof. Mahmoud Reza Aghamiri², Prof. Christian Kirisits ${ }^{3}, M S c$, PhD, \\ Reza Ghaderi, PhD² \\ 'Department of Radiotherapy, Tehran University of Medical Sciences, Tehran, Iran, ${ }^{2}$ Department of Medical Radiation Engineering, Shahid \\ Beheshti University, Tehran, Iran, ${ }^{3}$ Department of Radiotherapy and Oncology, Comprehensive Cancer Center, Medical University of Vienna, \\ Vienna, Austria
}

\begin{abstract}
Purpose: Intra-fractional organs at risk (OARs) deformations can lead to dose variation during image-guided adaptive brachytherapy (IGABT). The aim of this study was to modify the final accepted brachytherapy treatment plan to dosimetrically compensate for these intra-fractional organs-applicators position variations and, at the same time, fulfilling the dosimetric criteria.

Material and methods: Thirty patients with locally advanced cervical cancer, after external beam radiotherapy (EBRT) of 45-50 Gy over five to six weeks with concomitant weekly chemotherapy, and qualified for intracavitary high-dose-rate (HDR) brachytherapy with tandem-ovoid applicators were selected for this study. Second computed tomography scan was done for each patient after finishing brachytherapy treatment with applicators in situ. Artificial neural networks (ANNs) based models were used to predict intra-fractional OARs dose-volume histogram parameters variations and propose a new final plan.

Results: A model was developed to estimate the intra-fractional organs dose variations during gynaecological intracavitary brachytherapy. Also, ANNs were used to modify the final brachytherapy treatment plan to compensate dosimetrically for changes in 'organs-applicators', while maintaining target dose at the original level.

Conclusions: There are semi-automatic and fast responding models that can be used in the routine clinical workflow to reduce individually IGABT uncertainties. These models can be more validated by more patients' plans to be able to serve as a clinical tool.

Key words: ANN-based model, cervical cancer, IGABT, intra-fractional dose variations.

\begin{abstract}
Purpose
Combination of external beam radiotherapy (EBRT), brachytherapy, and concomitant chemotherapy was introduced as the standard treatment, for advanced cervical cancer patients [1]. Three-dimensional (3D) image-guided adaptive brachytherapy (IGABT) is an individually-optimized treatment for these patients [2,3]. Dose distribution optimization can be done by means of the initial, post-teletherapy, and pre-brachytherapy patients images, based on the GEC ESTRO (Groupe Européen de Curiethérapie European Society for Radiotherapy and Oncology) recommendations $[4,5]$.
\end{abstract}

Intra-fractional organs at risk (OARs) deformations that can lead to their dose variation during IGABT have been studied by other researchers. They reported these variations as one of the most important uncertainty factors of gynaecological (GYN) brachytherapy [6,7]. However, quality control of dose delivery in IGABT is an image-assistance procedure [8,9]. Treatment verification may be performed either by treatment planning systems (TPSs) or dedicated radiotherapy software (e.g., CERR or 3D Slicer) $[10,11]$. These dose delivery verifications approaches require registration of different patient image sets during brachytherapy procedure, which is tedious and time-consuming [12]. On the other hand, there
Address for correspondence: Zahra Siavashpour, PhD, Department of Medical Radiation Engineering, Shahid Beheshti University, Daneshjou BLV, Velenjak, Tehran, Iran, phone: +98 9120298 176,

e-mail: z_siavashpour@sbu.ac.ir, zahrasiavashpour@gmail.com
Received: 08.10.2017

Accepted: 20.12.2017

Published: 30.12 .2017 
is neither dose computing engine nor the ability to correct the treatment plan to adapt to the final anatomical situation, just before starting the procedure.

Treatment planning is a necessary step after each applicator insertion in IGABT due to the changes in relative position of OARs and CTVs to the applicators. Therefore, dose distribution of each brachytherapy fraction was assumed to be specific for that fraction. The hypothesis of this investigation is if one could modify this accepted plan to dosimetrically compensating the intra-fractional organs-applicators position variation, at the same time, fulfilling the dosimetric criteria.

Independently calculation of the magnitude of intrafractional OARs dose variations due to their deformations is desired in this study. Finally, fast and practical model to correct the treatment plan regarding the new OARs anatomical positions before dose delivery is the main purpose of the current study. This would lead to intra-fractional dose variations uncertainty reduction.

Artificial neural networks (ANNs), as an interesting branch of artificial intelligence (AI), have been developed enormously in medicine, and it is believed that they will have widespread applications and solutions to biomedical problems in the near future [13]. Already, they have been successfully applied to various medical areas, such as disease diagnosis, biochemical analysis, image analysis, and drug development [14]. ANNs are an information processing models that are composed of a large number of interconnected processing units (neurons) to resolve problems [14]. If the ANNs are trained properly, they will be able to generalize and quickly predict results for situations other than the training ones [15]. We tried to use this method to reach our aims during current study.

\section{Material and methods \\ Case selection criteria}

For this prospective study, 30 cervical cancer patients were selected. Patients' data were also previously used to assess the intra-fractional OARs dose variations by the same authors. Study was done at brachytherapy department of Atieh Hospital, Tehran, Iran, and the patients' inclusion criteria were: 1) locally advanced cervical cancer and after EBRT treatment of 45-50 Gy over five to six weeks with concomitant weekly chemotherapy $\left(50 \mathrm{mg} / \mathrm{m}^{2}\right.$ of cisplatin); 2) patient was qualified for intracavitary high-dose-rate (HDR) brachytherapy with tandem-ovoid applicators, i.e. no extended parametrial invasion distinguished by MRI. Patients' disease FIGO (International Federation of Gynecology and Obstetrics [16]) stages were IB1, IB2, and IIB, i.e. 5, 11, and 14 cases in each stage, respectively.

The study was approved by the institutional review board for the ethical rules considerations, and patients were informed about the study. A data workflow is presented in Figure 1, which summarized the study procedure to reach the mentioned aim.

\section{Patient preparation and pre-treatment steps}

Proper length and size of the Rotterdam tandem-ovoid applicators (Elekta AB, Stockholm, Sweden) were used for the patients under spinal sedation by a radiation oncologist in the operation room. The balloon of patient's bladder Foley catheter was filled with $6 \mathrm{~cm}^{3}$ of normal saline and $1 \mathrm{~cm}^{3}$ of meglumine compound (amp. VISIPAQUETM $320\left[50 \mathrm{~cm}^{3}\right]$, GE Healthcare, Ireland). Vaginal

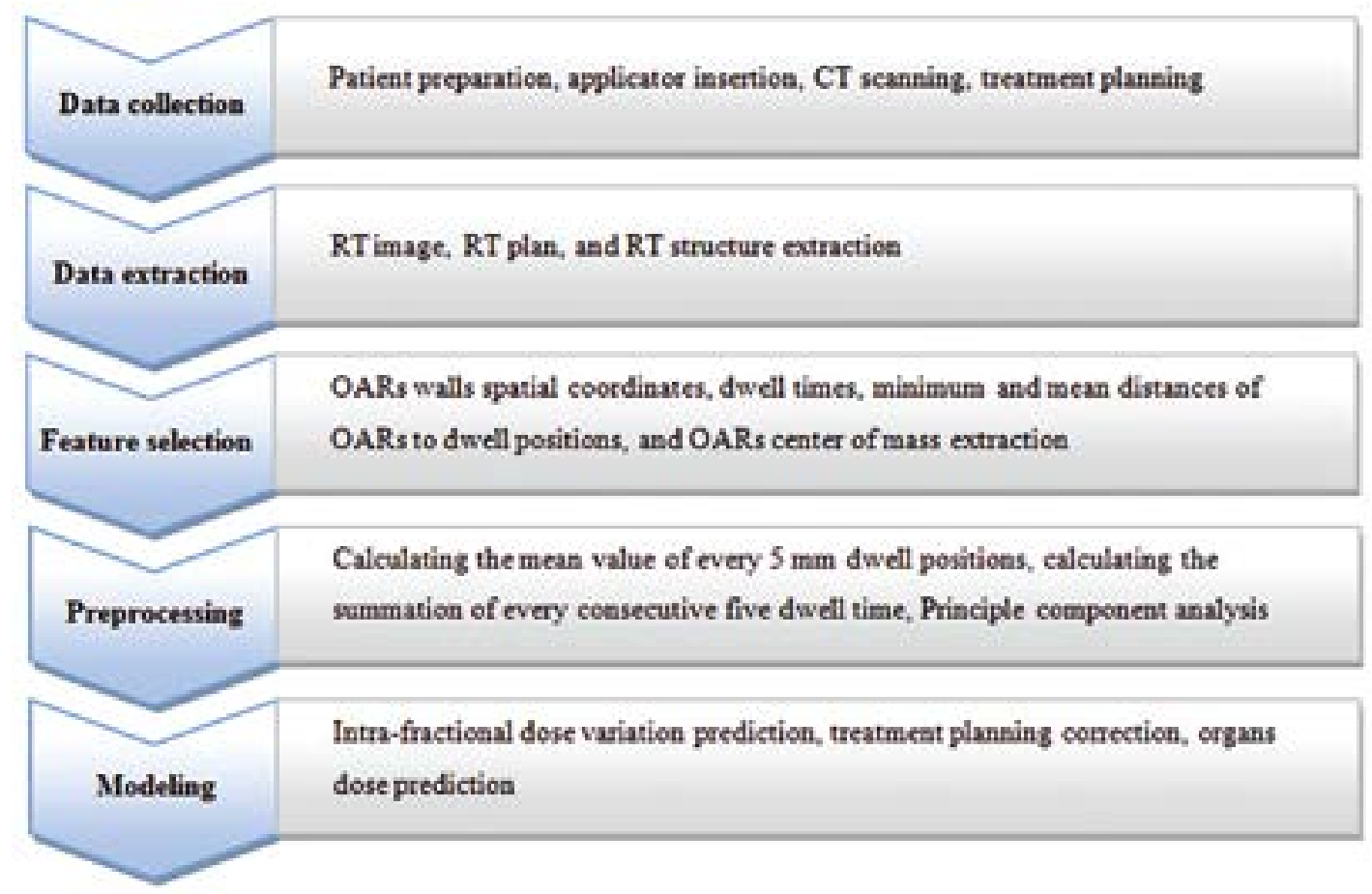

Fig. 1. Data workflow, which summarized the study approach 
packing was used to fix the applicators in place during the whole treatment.

After about half an hour after applicator insertion, patients were CT scanned (Somatom ${ }^{\circledR}$ DR Siemens scanner, Siemens Healthcare, Forchheim, Germany). Before scanning, $60 \mathrm{~cm}^{3}$ of normal saline was injected into the patient's bladder and it was clamped until the end of scanning.

Patients' CT images were imported to a 3D brachytherapy TPS (Flexiplan ${ }^{\circledR}$, version 2.6, isodose control, Elekta AB, Stockholm, Sweden). Intermediate-risk and high-risk clinical target volumes $\left(\mathrm{CTV}_{\mathrm{IR}}\right.$ and $\mathrm{CTV}_{\mathrm{HR}}$, respectively) were contoured by a radiation oncologist under the guidance of two MRIs; one at the time of diagnosis and one before the first brachytherapy fraction, based on the GEC-ESTRO recommendations [4,5,17]. Bladder, rectum, sigmoid, and OARs were delineated by the same physician. Applicators were reconstructed by the brachytherapy physicist.

Prescription dose was calculated based on the equivalent dose of $2 \mathrm{~Gy}$ per fraction $\left(\mathrm{EQD}_{2}\right)$ formula by considering $\alpha / \beta$ of $10 \mathrm{~Gy}$ for clinical target volumes (CTVs) and 3 Gy for OARs. Planning acceptance criteria were to deliver a minimum dose of $80-90$ Gy to $90 \%$ of the $\mathrm{CTV}_{\mathrm{HR}}$ volume $\left(D_{90}\right)$, and to keep the minimum dose to the most exposed $2 \mathrm{~cm}^{3}\left(\mathrm{D}_{2 \mathrm{~cm}^{3}}\right)$ of bladder less than $85 \mathrm{~Gy}$, and of the rectum and sigmoid 75 Gy $[5,18]$. Plans were optimized manually to reach the mentioned aims for each fraction. Important dose-volume histogram (DVH) parameters of CTVs (e.g., $\mathrm{D}_{90}, \mathrm{D}_{98}$, and the fractional target volume receiving $100 \%$ of the prescribed dose $\left[\mathrm{V}_{100}\right]$ ) and OARs (e.g., $\mathrm{D}_{2 \mathrm{~cm}^{3},} \mathrm{D}_{0.1 \mathrm{~cm}^{3}}, \mathrm{D}_{10}, \mathrm{D}_{30}$, and $\mathrm{D}_{50}$ ) were recorded [1,19].

Treatment afterloading system of our center was Flexitron ${ }^{\circledR}$ and HDR treatments were done by ${ }^{192}$ Ir Flexisource $^{\circledR}$ (Elekta AB, Stockholm, Sweden). Normal saline injection $\left(60 \mathrm{~cm}^{3}\right)$ and clamping the bladder with Foley catheter was also repeated just before treatment. Figure 2 is a flowchart describing the following steps of study in brief.

\section{Intra-fractional dose variations calculation by TPS}

Just after finishing the treatment, patients were transferred to the CT imaging room, and the entire previous patient's bladder preparation and scanning were repeated without removing the applicators. Patient's movements were tried to be minimized by using a hard backboard. Time duration between finishing the treatments and second CT scans was about 25 minutes. Post-treatment CT images were also imported to the TPS. Inter-observer uncertainty of $9 \%$ (target) and 5-11\% (OARs) were reported for organs delineation based on the Tanderup et al. study [20]. Therefore, all CTVs and OARs were delineated by an experienced oncologist. That would lead to a similar uncertainty for all the cases. Consequently, when we calculated the intra-fractional variations, the impact of these intra-observer uncertainties and their influence on obtained results could be considered insignificant. We had to delineate CTVs on the second image series because our treatment planning system didn't have deformable registration ability. Treatment plans were copied man- ually to the second image series. New DVH parameters were recorded for OARs, and their intra-fractional dose variations were calculated as completely explained in the published paper [12]. The time required for calculating these organs dose variations of each patient was about 45 minutes.

\section{Intra-fractional dose variations prediction modeling}

CT image series, delineated organ structures, and planning data of each pre- and post-treatment plan were exported from TPS in DICOM format. Also, source dwell times were extracted from plans reports. Following features were extracted from these files by in-house MATLAB codes; these features were further used in intra-fractional OARs dose variations prediction modeling (Figure 2, step 1): 1) the minimum distance of source dwell positions along the applicators to the OARs' walls (defined as D); spatial coordinates of OARs and applicators walls were exported from RT-structure files; 2) the mean distance of CTVs' walls to the source dwell positions (again defined as D, but now for targets); 3) the product of dwell times and source activity at the treatment time (defined as TA); 4) the product of $\frac{T}{d^{2}}$ and source strength at the treatment time for every source dwell positions along the applicators (defined as TDA), where $\mathrm{d}$ is the minimum distance of each dwell position to the OARs wall and $\mathrm{T}$ is its source dwell time; 5) the mean distance of each source dwell positions to the OARs wall (defined as MD); 6) the distance of each source dwell position to each OARs center of mass (defined as COM).

\section{Artificial neural networks}

The main aim of this study was to correct a treatment plan, so we faced to an inverse problem that has a complicated nature and cannot be solved be a routine theoretical or analytical formula. Also, the characteristic of the issue was such that the data dimension was high and we didn't have any prior knowledge of relationships between changes in organs location and the resultant dose variations. Therefore, simple statistical analysis methods were inefficient, and we had to use ANNs to reach our aims and solve this inverse problem.

Multilayer perceptron (MLP) and radial basis functions (RBF) are the networks we evaluated and their prediction ability. MLP's objective is to explain a model that maps sets of input data onto a set of appropriate outputs, so that the model can generate the output when the preferred output is unknown [21,22]. MLP utilizes back-propagation algorithm (BPA) for training. The parameters that must be determined were the number of hidden layer and the neurons in each layer. Error selection should be in such a way that generalization of designed network would be preserved [23].

RBF network uses radial basis functions as activation functions. Determination of centers and spread of activation functions were done by the complete interpolation method and manual specification proportional to our data spread, respectively [24]. RBF networks have relatively better performance than the MLPs (problems with strong local characteristics and small training data set 


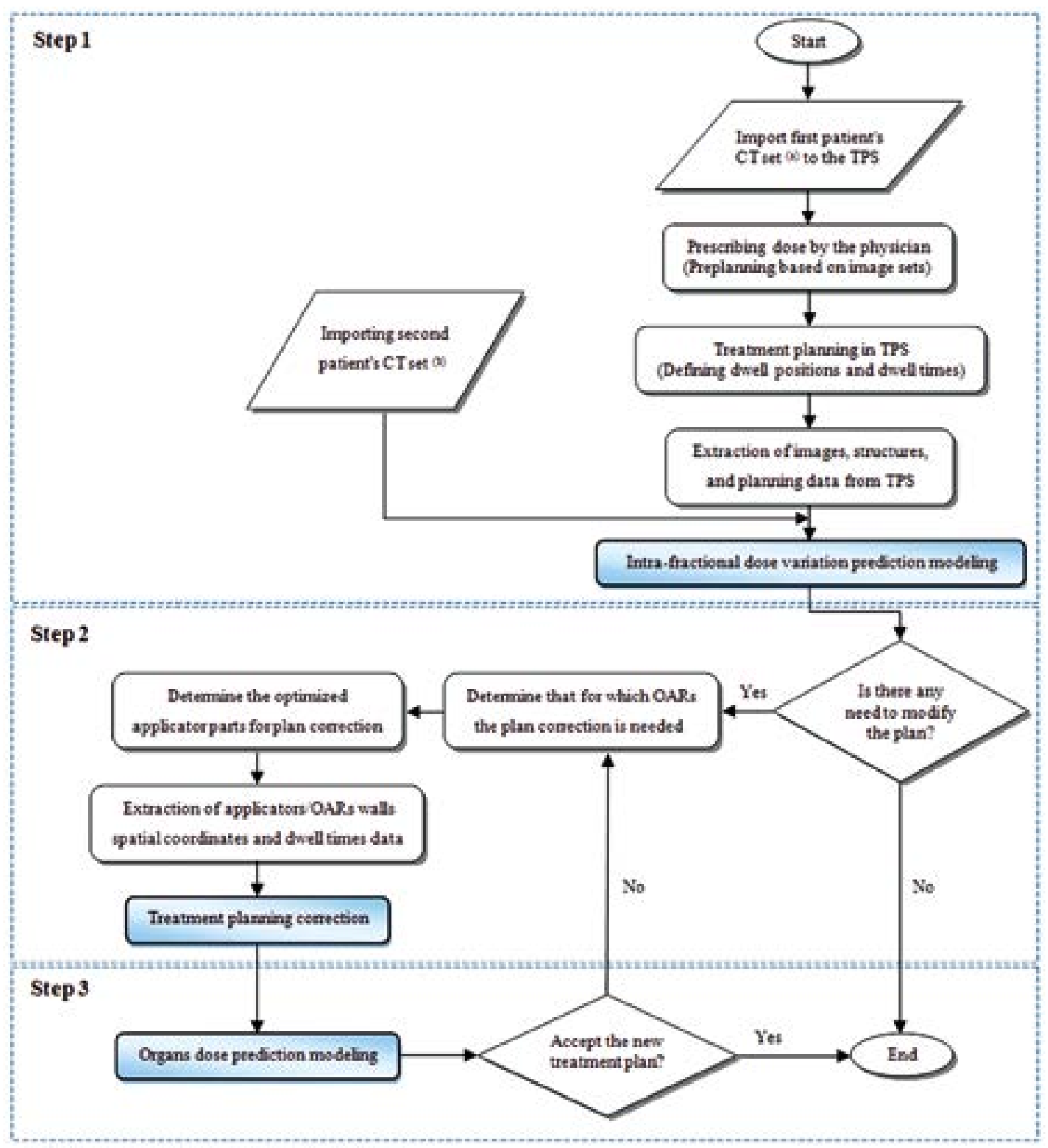

(a) CT sets obtained for treatment planingpuppose

(b) CT sets oteained for qualiry control of dose dellvery and checking for any otgan's intra-fractisnal variabsas

Fig. 2. Flowchart describing different steps of study

size) $[25,26]$. The objective function in our learning process was to minimize the mean percentage error (MPE) of these learning algorithms.

\section{Preprocessing phases of data}

Our treatment planning was based on allocating source dwell times to the appropriate dwell positions continuously (every $1 \mathrm{~mm}$ ). Therefore, input features were extracted for every $1 \mathrm{~mm}$ across the source paths by means of in-house written codes. To overcome the complexity of designed models, obtained distances were averaged and dwell times were summed for every $5 \mathrm{~mm}$ along each applicator. This idea is not far-fetched by considering the discrete source steps planning technique that is being used in lots of brachytherapy centers. Obtained data were normalized because organs to applicators distances included a large range of numbers.

We also tested the principal components analysis (PCA) method as a data pre-processing. By PCA, we could guess the data patterns and compressed them 
without much loss of information [27]. The proportionality of the data was evaluated by the Kaiser-Meyer-Olkin (KMO) test and Bartlette's test of sphericity. Communalities of data were checked to examine if there were any outlier points. Finally, we determined how many factors were required for each organ to represent as their principle component.

We had access to a limited patients' data. Also, we didn't use the entire data for model training to avoid the over fitting problem. A better approach is to split the data into disjoint subsets: training set, used to train the model; test set, used to estimate the error of the trained model. Therefore, 20 cases were chosen for training and the other 10 for testing the model. The justification of this data categorization was tested by 20 times choosing them randomly, performing the models, and obtaining their MPE and its standard deviation.

Furthermore, leave one out (LOO) cross-validation also examined to overcome the probability of data insufficiency during each modeling. LOO cross-validation for a dataset with $\mathrm{N}$ examples performs $\mathrm{N}$ experiments, for each experiment use $\mathrm{N}-1$ examples for training and the remaining example for testing.

\section{Treatment planning correction}

After detection of intra-fractional organs deformations and their resulted changes on DVH parameters, it was time to ask if the plan correction is necessary ('YES') or not $\left({ }^{\prime} \mathrm{NO}^{\prime}\right)$. It is the physician responsibility to answer this question. If the response was 'YES', the next step would start (Figure 2, step 2). The accepted plan was assumed to be the ideal plan. Therefore, we tried to reach almost the same OARs DVH parameters to this plan, simultaneous with obeying the CTVs dose criteria. We used both the plan's extracted data of applicators/OARs walls spatial coordinates and source dwell times for plan correction. New source dwell times were proposed for the planned dwell positions regarding the TG43 algorithm (which is based on 'inverse square law') and using the aforesaid extracted data [28]. This enabled us to calculate a final treatment plan for each patient to fit with the patient's OARs new anatomical location. Following formulas were used to find the new dwell times:

$$
\frac{\frac{T_{A}}{d_{A}^{2}}}{\frac{T_{B}}{d_{B}^{2}}}=1 \quad \Rightarrow \frac{T_{A}}{d_{A}^{2}}=\frac{T_{B}}{\frac{d_{B}^{2}}{2}} \Rightarrow T_{A}=\frac{T_{B}}{d_{B}^{2}} \times \overline{d_{A}^{2}}
$$

where $T_{A}$ and $T_{B}$ are dwell time of the first treatment plan and dwell time calculated to compensate organs deformations, respectively. We considered each dwell time to be zero if $T_{B}-T_{A}$ became negative. We tried to consider dwell positions, in which we allocated times in the first plan.

It was a long-term process when we searched through the whole applicators length to compensate each OARs dose variation. Therefore, we decided to temporally optimize this searching step. Figure 3 is a flowchart demonstrating different steps tested to determine the optimum applicators parts for treatment plan correction regarding each OARs.
Note, that we just tried to correct the organ's dose for which their intra-fractional variations were higher than $5 \%$ of accepted plan. Treatment plans were corrected in the TPS after calculating the $\mathrm{T}_{\mathrm{A}} \mathrm{s}$. $\mathrm{D}_{90}$ of $\mathrm{CTV}_{\mathrm{IR}}$, and $\mathrm{CTV}_{\mathrm{HR}}$ were recorded after plan corrections. Criteria for accepting the new plans were: 1 - if OARs' dose $\left(D_{2 \mathrm{~cm}^{3}}\right)$ be as near as possible (less than $\pm 5 \%$ difference) to the first plan; 2 - if $\mathrm{D}_{90}$ of $\mathrm{CTV}_{\mathrm{IR}}$ and $\mathrm{CTV}_{\mathrm{HR}}$ be more than $70 \mathrm{~Gy}$ and $87 \mathrm{~Gy}$ (in EQD2) for total patient treatment, respectively.

\section{Organs dose prediction modeling}

Manually correcting the plan in TPS was a time-consuming process. Therefore, we tried to design another ANN model that can predict organs and targets DVH parameters after getting dwell times and organs-to-applicators distance data (Figure 2, step 3). All data of sixty cases (both before and after treatment images and planning data) were used, independently. Input features were the same as explained for first models. However, here the outputs were the net DVH parameters (not their difference) of OARs $\left(\mathrm{D}_{2 \mathrm{~cm}^{3},}, \mathrm{D}_{0.1 \mathrm{~cm}^{3}}\right.$ as hot points, and $\mathrm{D}_{10}$ $\mathrm{D}_{30}$, and $\mathrm{D}_{50}$ as mean dose) and CTVs $\left(\mathrm{D}_{90}\right.$ and $\left.\mathrm{D}_{98}\right)$ for each case. MLP and RBF models were designed. Fifty cases were chosen for training and the last 10 for testing, randomly. Moreover, PCA and LOO preprocessing techniques were tested in these models.

\section{Results \\ Intra-fractional OARs dose variations}

TPS-based intra-fractional OARs dose variations are presented in Table 1. The time required for calculating these dose variations was about 45 minutes for each patient [12].

\section{Pre-processing of data}

As it was mentioned before, explained features were extracted from the DICOM files for every $5 \mathrm{~mm}$ of applicators active length; e.g., for a tandem with $7 \mathrm{~cm}$ active length we had 14 data of each features. Therefore, to have the same matrix size for each patient's input features, we considered a matrix with 30 rows ( 20 for tandem and 5 for each ovoid data).

The reliability of applying the PCA method to reduce the data dimension was satisfied by KMO and Bartlett's tests, e.g., for bladder KMO statistical result was 0.703 (which is $>0.5$ ), and significance of Bartlett's test was 0.00 (which is < 0.05). Communalities of data were obtained suitable (communality coefficients were $>0.5$ ) and there was no need to omit any variable. Finally, we got 7, 8, 8, 8 , and 9 factors to almost completely explain the bladder, rectum, sigmoid, $\mathrm{CTV}_{\mathrm{HR}}$, and $\mathrm{CTV}_{\mathrm{IR}}$ data variances, respectively.

For MLP networks, a resilient back-propagation algorithm (Rprop) was used due to higher performance speed and non-interference in results of the network. Centers selection was done based on the complete data interpolation in RBF networks. Networks spreads were chosen manually based on the data extension. 


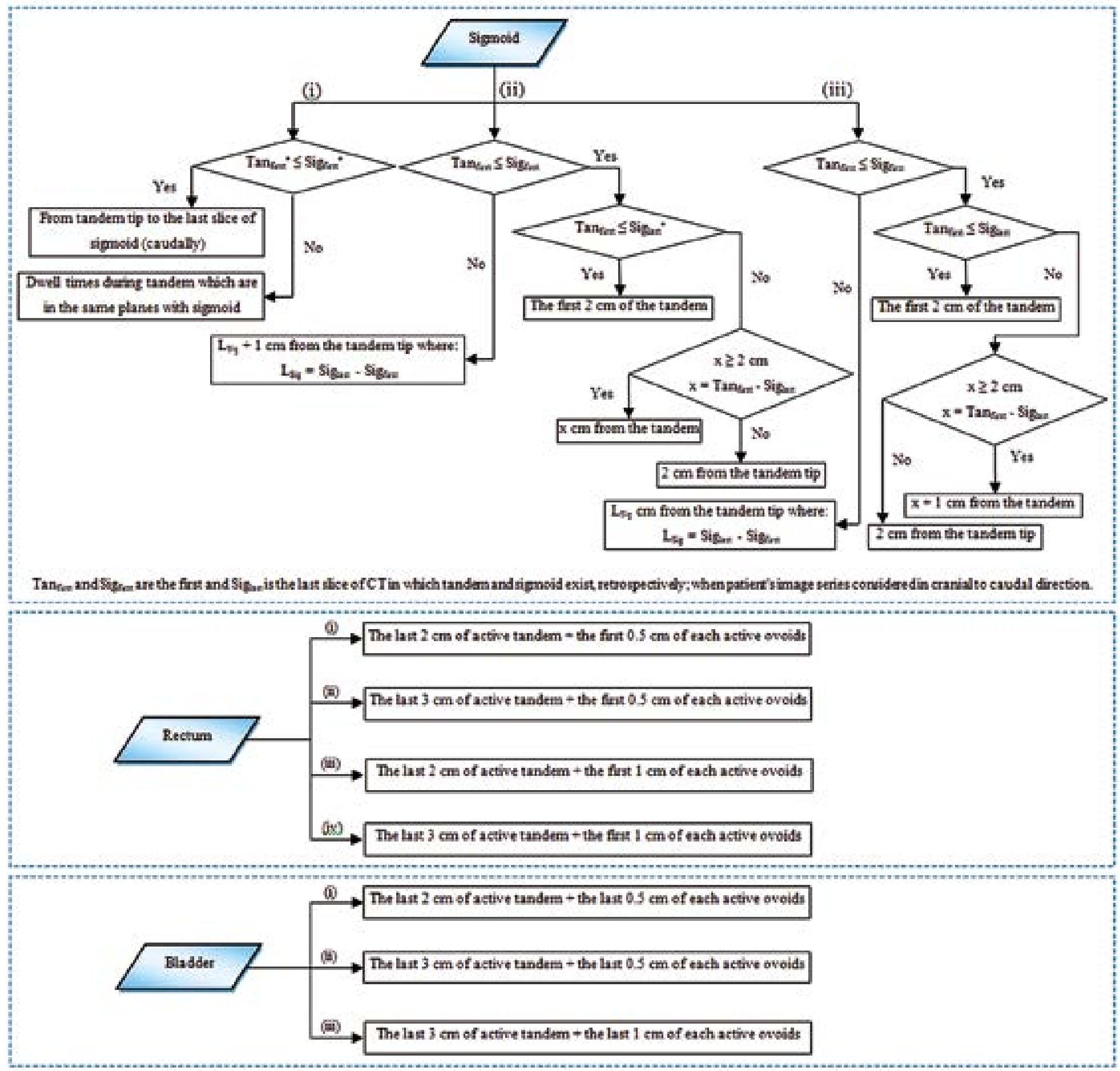

Fig. 3. Flowchart demonstrating steps tested to determine optimized applicators parts for treatment plan correction

Networks for OARs intra-fractional dose variations prediction

Data of 30 paired-cases were available. Input features contained TA, D, TDA, MD, and COMD difference of preand post-treatment data. Outputs were the variations of organs DVH parameters during a treatment course.

We tested our MLP models generalizability by 20 times performing them with different 20 cases random se- lection as train and 10 for test sets, whenever the LOO technique was not practical. The standard deviations of obtained MPE were $0.12,0.13,0.17,2.0$, and 0.19 for bladder, rectum, sigmoid, $\mathrm{CTV}_{\mathrm{HR}}$, and $\mathrm{CTV}_{\mathrm{IR}}$, respectively. Also, the tested number of hidden layers and the number of neurons in each layer were 2 to 5 , and 5 to 30 , respectively. The best results were obtained when five hidden layers were designed, without using the LOO method. 
Table 1. Intra-fractional organs at risk (OARs) relative dose variations (mean values averaged over 30 patients) [9]. Percentage of dose-volume histogram (DVH) parameters variations (mean $\pm \mathrm{SD})(\%)$

\begin{tabular}{lcccccc} 
OARs & $\mathrm{D}_{2 \mathrm{~cm}^{3}}$ & $\mathrm{D}_{0.1 \mathrm{~cm}^{3}}$ & $\mathrm{D}_{10}$ & $\mathrm{D}_{30}$ & $\mathrm{D}_{50}$ & \% Organ volume change \\
\hline Bladder & $-2.9 \pm 18.7$ & $-6.3 \pm 26.4$ & $1.7 \pm 14.0$ & $4.1 \pm 12.9$ & $4.0 \pm 13.8$ & $-13.0 \pm 36.4$ \\
\hline Rectum & $0.3 \pm 17.8$ & $1.3 \pm 21.6$ & $2.3 \pm 14.5$ & $2.8 \pm 13.1$ & $3.8 \pm 14.3$ & $-5.8 \pm 19.4$ \\
\hline Sigmoid & $-2.1 \pm 17.3$ & $-3.6 \pm 18.3$ & $-2.7 \pm 14.2$ & $-2.5 \pm 14.0$ & $-4.1 \pm 17.3$ & $1.4 \pm 27.0$
\end{tabular}

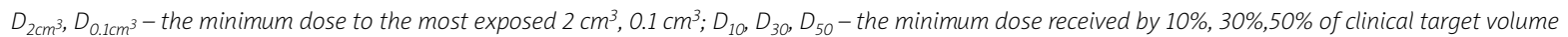
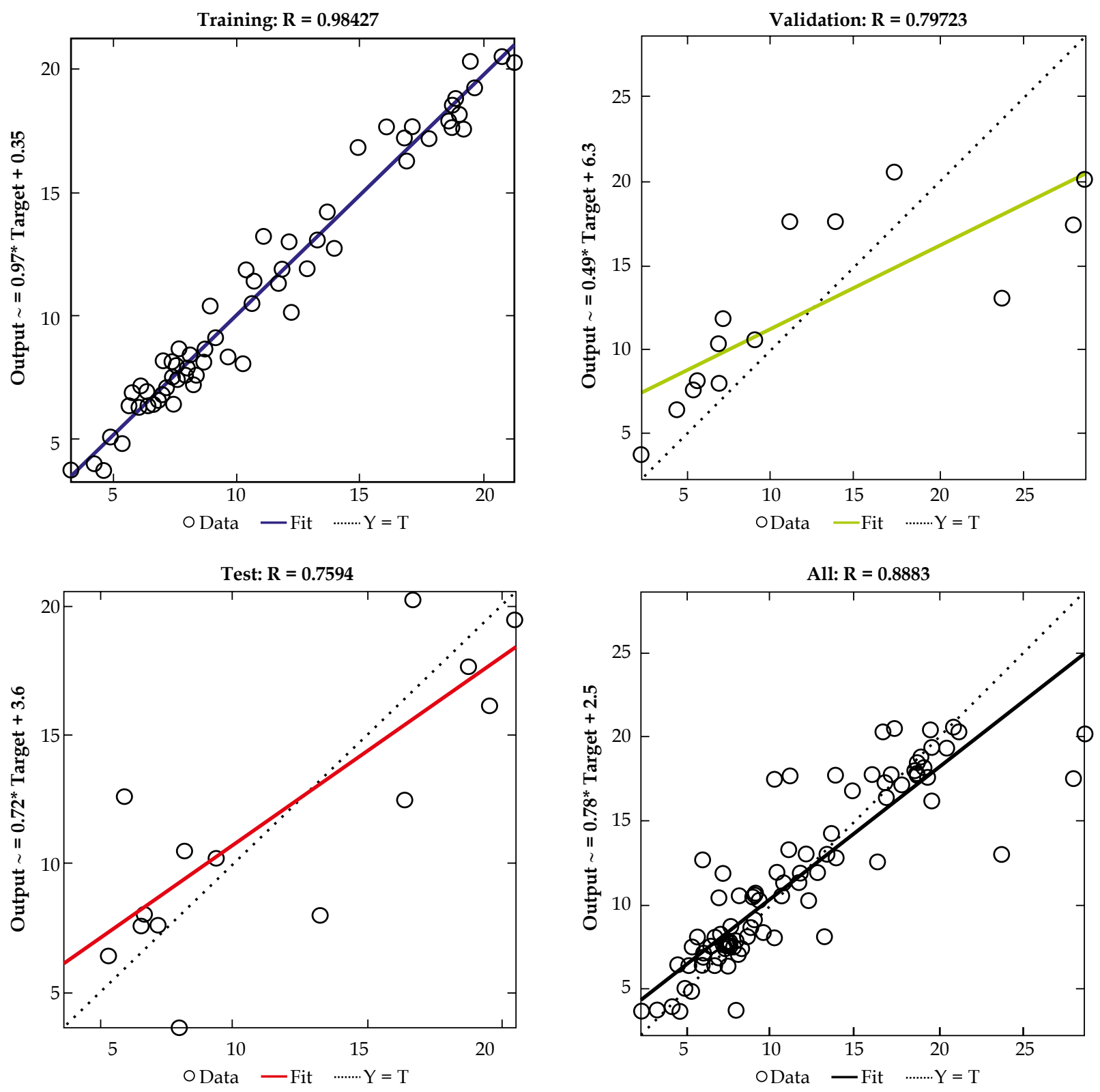

Fig. 4. An example of a regression plots for an multilayer perceptron network designed to predict intra-fractional organs at risk dose variations. The four plots represent the training, testing, validation, and all data. Dashed line of each plot represents result - outputs $=$ targets

Each network was conducted with three runs, and standard deviations of obtained MPEs were calculated as presented in Table 2.

Network validation was tested by creating regression plot, which shows the relationship between the pre- dicted outputs and the targets (Figure 4). If the network training was perfect, the network outputs and the targets would be exactly equal, but that is generally a rare case. The solid line represents the best fit linear regression line between outputs and targets [29]. 
For RBF networks spread and number of neurons were tried between 50 to 200 , and 5 to 30 , respectively. LOO technique did not lead to better result, except for sigmoid and $\mathrm{CTV}_{\mathrm{HR}}$. The performance of designed networks were analyzed by mean square error (MSE) (Figure 5) [29]. Results and characteristics of designed MLP and RBF networks are presented in Table 2.

\section{Treatment plans correction}

Generally, of the 30 patients, just 10, 14, and 12 cases needed bladder, rectum, and sigmoid dose correction, respectively. Therefore, new source dwell times were calculated. Most sensitive organs at risk (i.e., sigmoid and rectum) had priority in situation where two organs dose musted be corrected (Figure 2). Tables 3-5 present outputs of TPS after applying new dwell times.

\section{Networks for organs dose prediction}

Data of 60 cases were independently considered. In this step, input features were contains net TA, D, TDA, $\mathrm{MD}$, and COMD. Networks output for each case were organ's DVH parameters.

For MLP networks, number of hidden layers were tested, and number of neurons in each layer were 2 to 5 , and 5 to 50 , respectively. Also, RBF network spread and number of neurons were examined between 50 to 200, and 5 to 50 , respectively. Results and characteristics of MLP and RBF networks are presented in Table 6. LOO technique gave better results for rectum, and $\mathrm{CTV}_{\mathrm{IR}}$ in MLP, and bladder and $\mathrm{CTV}_{\mathrm{IR}}$ in RBF networks.

\section{Discussion}

Intra-fractional organs deformations can be named as a major source of uncertainties during HDR ${ }^{192}$ Ir intracavitary brachytherapy for cervical cancer patients $[6,30,31]$. Online treatment planning correction is reasonable based on these days' improvements on image guidance in brachytherapy rooms $[32,33]$. Some fast intra/interorgan dose variations calculations methods were already proposed using radiotherapy research toolkits such as CERR and 3D Slicer [10]. However, none of these softwares claimed to correct the plans; therefore, they could just give a report of occurred variations. Also, it will be hard to compensate these variations in the subsequent treatment course due to the high 'planning aim dose' per fraction.

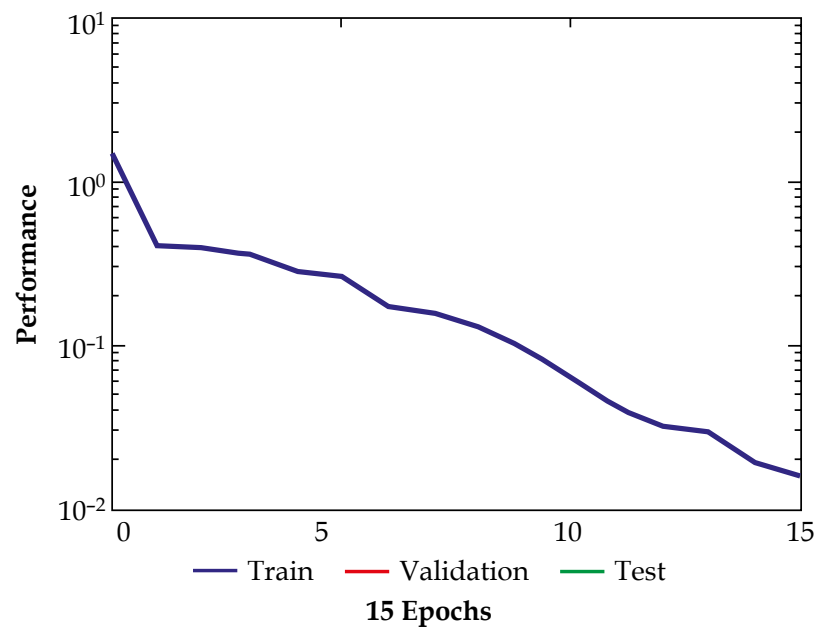

Fig. 5. An example of performance (mean square error MSE) plot for an radial basis functions network designed to predict intra-fractional organs at risk dose variations

Table 2. Characteristics and performance results of multi-layer perceptron (MLP) and radial basis functions (RBF) networks for prediction the organs at risk (OARs) intra-fractional dose variations

\begin{tabular}{|c|c|c|}
\hline Organs & $\begin{array}{c}\text { MLP network } \\
\text { (\# of layers } \\
\text { [\# of neurons in each layer]) } \\
\text { MPE } \% \pm \text { SD } \%\end{array}$ & $\begin{array}{c}\text { RBF network } \\
\text { (\#) neurons, spread) } \\
\text { MPE \% }\end{array}$ \\
\hline Bladder & $\begin{array}{c}(5[20,20,20,10,5]) \\
11.0 \pm 2.1\end{array}$ & $\begin{array}{c}(10,100) \\
6\end{array}$ \\
\hline Rectum & $\begin{array}{c}(5[30,20,10,5,5]) \\
9.0 \pm 0.6\end{array}$ & $\begin{array}{c}(5,50) \\
5\end{array}$ \\
\hline Sigmoid & $\begin{array}{c}(5[30,20,10,5,5]) \\
15.0 \pm 1.2\end{array}$ & $\begin{array}{c}(5,150) \\
8\end{array}$ \\
\hline $\mathrm{CTV}_{\mathrm{IR}}$ & $\begin{array}{c}(5[20,10,10,5,5]) \\
10.0 \pm 1.0\end{array}$ & $\begin{array}{c}(10,200) \\
7\end{array}$ \\
\hline $\mathrm{CTV}_{\mathrm{HR}}$ & $\begin{array}{c}(5[30,10,10,5,5]) \\
14.0 \pm 2.6\end{array}$ & $\begin{array}{c}(5,150) \\
10\end{array}$ \\
\hline
\end{tabular}

MPE - mean percentage error, CTV - clinical target volume, CTVHR - high-risk clinical target volume, CTVIR - intermediate-risk clinical target volume

We designed a pilot study with 30 independent statistical populations. Intra-fractional dose variations during one of their brachytherapy fractions were calculated to justify the suitability of selected cases for the following

Table 3. Bladder dose correction results after applying new dwell time

\begin{tabular}{|c|c|c|c|c|c|c|c|c|c|c|}
\hline \multirow[t]{3}{*}{ Conditions for bladder } & \multicolumn{9}{|c|}{ Number of cases (from 10 total cases) } & \multirow[t]{3}{*}{ Accept } \\
\hline & \multicolumn{3}{|c|}{$\mathrm{D}_{90} \mathrm{HR}$} & \multicolumn{3}{|c|}{$\mathrm{D}_{90} \mathrm{IR}$} & \multicolumn{3}{|c|}{$\mathrm{D}_{2 \mathrm{~cm}^{3}}$ (bladder) } & \\
\hline & Pass & Fail & NAN & Pass & Fail & NAN & Pass & Fail $^{*}$ & NAN & \\
\hline (i) & 6 & 0 & 4 & 6 & 0 & 4 & 0 & 6 & 4 & \\
\hline (ii) & 9 & 1 & 0 & 9 & 1 & 0 & 6 & 4 & 0 & $\leftarrow$ \\
\hline (iii) & 9 & 1 & 0 & 7 & 3 & 0 & 7 & 3 & 0 & \\
\hline
\end{tabular}

Pass - organs at risk intra-fractional variation became less than 5\%, Fail - organs at risk intra-fractional variation remain higher than $5 \%$, NAN - no data (dwell times) was available, $D_{90}$-the minimum dose received by $90 \%$ of clinical target volume, $D_{2 \mathrm{~cm}^{3}}$ - the minimum dose to the most exposed $2 \mathrm{~cm}^{3}$, HR - high-risk, $I R$-intermediate-risk clinical target volume

*Two cases of four failed one intra-fractional variation and became about $7.5 \%$ from $17.5 \%$ 
Table 4. Rectum dose correction results after applying new dwell time

\begin{tabular}{|c|c|c|c|c|c|c|c|c|c|c|}
\hline \multirow[t]{3}{*}{ Conditions for rectum } & \multicolumn{9}{|c|}{ Number of cases (from 12 total cases) } & \multirow[t]{3}{*}{ Accept } \\
\hline & \multicolumn{3}{|c|}{$\mathrm{D}_{90} \mathrm{HR}$} & \multicolumn{3}{|c|}{$\mathrm{D}_{90} \mathrm{IR}$} & \multicolumn{3}{|c|}{$\mathrm{D}_{2 \mathrm{~cm}^{3}}($ rectum $)$} & \\
\hline & Pass & Fail & NAN & Pass & Fail & NAN & Pass & Fail & NAN & \\
\hline (i) & 8 & 0 & 4 & 8 & 0 & 4 & 3 & 5 & 4 & \\
\hline (ii) & 9 & 0 & 3 & 9 & 0 & 3 & 4 & 5 & 3 & \\
\hline (iii) & 9 & 0 & 3 & 9 & 0 & 3 & 7 & 2 & 3 & $\leftarrow$ \\
\hline (iv) & 11 & 0 & 1 & 11 & 0 & 1 & 10 & 1 & 1 & \\
\hline
\end{tabular}

Pass - organs at risk intra-fractional variation became less than 5\%, Fail - organs at risk intra-fractional variation remain higher than $5 \%$, NAN - no data (dwell times) was available, $D_{90}$ - the minimum dose received by $90 \%$ of clinical target volume, $D_{2 \mathrm{~cm}^{3}}$ - the minimum dose to the most exposed $2 \mathrm{~cm}^{3}$, HR - high-risk, $\mathbb{I R}$ - intermediate-risk clinical target volume

Table 5. Sigmoid dose correction results after applying new dwell time (TAs) Conditions for sigmoid Number of cases (from 12 total cases)

\begin{tabular}{lcccccccccc} 
& \multicolumn{4}{c}{$\mathrm{D}_{90} \mathrm{HR}$} & \multicolumn{4}{c}{$\mathrm{D}_{90} \mathrm{IR}$} & \multicolumn{2}{c}{$\mathrm{D}_{2 \mathrm{~cm}^{3}}$ (sigmoid) } \\
\cline { 2 - 14 } & Pass & Fail & NAN & Pass & Fail & NAN & Pass & Fail & NAN \\
\hline (i) & 10 & 0 & 2 & 10 & 0 & 2 & 4 & 6 & 2 \\
\hline (ii) & 10 & 0 & 2 & 10 & 0 & 2 & 5 & 7 & 0 & \\
\hline (iii) & 11 & 1 & 0 & 12 & 0 & 0 & 8 & $4^{*}$ & 0 & $\leftarrow$
\end{tabular}

Pass - organs at risk intra-fractional variation became less than 5\%, Fail - organs at risk intra-fractional variation remain higher than $5 \%$, NAN - no data (dwell times) was available, $D_{90}$-the minimum dose received by $90 \%$ of clinical target volume, $D_{2 \mathrm{~cm}^{3}}$ - the minimum dose to the most exposed $2 \mathrm{~cm}^{3}$, HR - high-risk, $I R$-intermediate-risk clinical target volume

${ }^{\star}$ For 4 failed cases, sigmoid had about $26 \%$ dose reduction after applying the new dwell times, even with the intra-fractional variations still higher than $5 \%$

Table 6. Characteristics and performance results of multi-layer perceptron (MLP) and radial basis function (RBF) networks for organs dose prediction

\begin{tabular}{|c|c|c|}
\hline Organs & 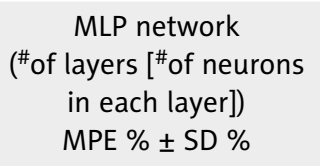 & $\begin{array}{l}\text { RBF network } \\
\text { (\# of neurons, } \\
\text { spread) } \\
\text { MPE \% }\end{array}$ \\
\hline Bladder & $\begin{array}{c}(2[40,10]) \\
18.0 \pm 3.1\end{array}$ & $\begin{array}{c}(40,150) \\
5\end{array}$ \\
\hline Rectum & $\begin{array}{c}(4[30,20,10,10]) \\
21.0 \pm 2.3\end{array}$ & $\begin{array}{c}(25,150) \\
6\end{array}$ \\
\hline Sigmoid & $\begin{array}{c}(3[30,20,10]) \\
28.0 \pm 2.1\end{array}$ & $\begin{array}{c}(25,150) \\
6\end{array}$ \\
\hline $\mathrm{CTV}_{\mathbb{I R}}$ & $\begin{array}{c}(5[20,10,10]) \\
20.0 \pm 0.6\end{array}$ & $\begin{array}{c}(25,150) \\
9\end{array}$ \\
\hline $\mathrm{CTV}_{\mathrm{HR}}$ & $\begin{array}{c}(5[40,20,10,10,5]) \\
22.0 \pm 2.5\end{array}$ & $\begin{array}{c}(20,150) \\
11\end{array}$ \\
\hline
\end{tabular}

$M P E$ - mean percentage error, $C T V_{H R}$ - high-risk clinical target volume, $C T V_{I R}$ - intermediate-risk clinical target volume

steps. Intra-fractional dose variations that we obtained (Table 1) was in the similar range of previous investigators results, in one applicator insertion for several treatment fractions' protocol [34]. The magnitude of random errors (i.e., standard deviations) is a good reason for thinking about a correction solution.

A definition developed in 1980s named the 'instant physician', meaning that a neural network trains automatically by a lot of medical information records (i.e., symptoms, diagnosis, and treatment) for each particular patient. After training, by introducing a new case symptom, the network is expected to guess the 'best' diagnosis and its treatment [35]. In the current study, we suggested repeating the procedure only by introducing a model, which can get the intra-fractional OARs dose variations and suggest the best corrected-plan to reach the treatment aims. This model can be called as 'instant physicist'.

Neural networks were used in radiotherapy physics domain for pre-planning optimization, treatment plan optimization, and plans scoring and evaluations $[15,36,37]$. In this pilot study, we used neural networks and optimized their architectures to minimize intra-fractional organs dose variations during a GYN intracavitary brachytherapy course. Neural networks were chosen to develop our model due to the high complexity of our problem.

RBF network can be used in non-parametric models, and its primary goal is to estimate underlying function or output of desired input values [38]. These networks were more applicable to our issues, both for intra-fractional dose variations predictions and also planning outputs estimations after applying new dwell times (Tables 2 and 5). Networks results for $\mathrm{CTV}_{\mathrm{IR}}$ and $\mathrm{CTV}_{\mathrm{HR}}$ were expectable due to high gradient of brachytherapy dose; these organs are located in high dose regions, and are very sensitive to any small inaccuracy in data extraction and networks limitations.

Proposing new dwell times needed to search along the applicators active length, which took a long time, about $20 \pm 4$ minutes for studied cases with a core i7 system, $\mathrm{CPU}=2 \mathrm{GHz}$, and RAM $=8 \mathrm{~GB}$. By applicator segmentation and considering defined partitions for each OARs, this executing time reduced to $6 \pm 1$ minute. Applicators partition selections were based on our treatment planning experiences and manual optimizations. For bladder dose correction, the best part of applicators was the lower 
$3 \mathrm{~cm}$ and $0.5 \mathrm{~cm}$, and for rectum were the lower $2 \mathrm{~cm}$ and $1 \mathrm{~cm}$ of tandem and each ovoid active length, respectively. For sigmoid, it depends on its relative position to the tandem, and almost considering 2 to $3 \mathrm{~cm}$ of its tip dwell positions as rational. More accurate applicator partitioning may also be done by a genetic algorithm.

This was a pilot study, and an investigation with a larger statistical population may lead to a more accurate model and optimum ANN architecture. Also, using a larger dataset will allow to extend the proposed model to a wider range of brachytherapy treatments, even with other applicator types. As a final note, the presented approach is capable of speeding up the plan optimization and can be introduced as a module to the TPSs in the routine clinical workflow. The model will be faster and user-friendly if the organs segmentation is done automatically.

To summarize, we investigate the intra-fractional OARs dose variations occurring due to their deformation. An independent model was designed, which is able to semi-automatically extract the treatment planning data from TPS, and calculate OARs DVH variations and asking physicians if any change is required. If the answer is 'YES', the model will try to compensate the variations by manipulating the plans parameters (dwell times), and finally estimate a new organs DVH parameters. Time optimization was done in searching for the relevant dwell positions along the applicators. This optimization will guarantee the practicality of proposed models based on routine clinical workflow. Plan correction will be done depending of the planning aim criteria. The validity of the proposed models were established by a commercial TPS.

\section{Conclusions}

A tailored ANN-based model was developed to estimate the intra-fractional organs dose variations during intracavitary GYN brachytherapy. Also, another model was designed to modify the final brachytherapy treatment plans to compensate dosimetrically for intra-fractional changes in 'organs-applicators' relative position, while maintaining target dose and OARs DVH parameters at the original plan level. There are semi-automatic and fast responding models that can be used in the routine clinical workflow to reduce the IGABT uncertainties, individually. These models can be more generalized and validated additionally by more patients' plans, ideally from other institute, to be able to serve as a clinical tool incorporated by commercial TPSs.

\section{Disclosure}

Authors report no conflict of interest.

\section{References}

1. ICRU/GEC-ESTRO report no. 89. Prescribing, Recording and Reporting Brachytherapy for Cancer of the Cervix. International Commission on Radiation Units \& Measurements, Inc. 2016.

2. Wang $X$, Li J, Wang $P$ et al. Image-guided radiation therapy boost in combination with high-dose-rate intracavitary brachytherapy for the treatment of cervical cancer. J Contemp Brachytherapy 2016; 8: 122-127.
3. Otter S, Franklin A, Ajaz M et al. Improving the efficiency of image guided brachytherapy in cervical cancer. J Contemp Brachytherapy 2016; 8: 557-565.

4. Haie-Meder C, Pötter R, Van Limbergen E et al. Recommendations from Gynaecological (GYN) GEC-ESTRO Working Group (I): concepts and terms in 3D image based 3D treatment planning in cervix cancer brachytherapy with emphasis on MRI assessment of GTV and CTV. Radiother Oncol 2005; 74: 235-245.

5. Pötter R, Haie-Meder C, Van Limbergen E et al. Recommendations from gynaecological (GYN) GEC ESTRO working group (II): concepts and terms in 3D image-based treatment planning in cervix cancer brachytherapy-3D dose volume parameters and aspects of 3D image-based anatomy, radiation physics, radiobiology. Radiother Oncol 2006; 78: 67-77.

6. Simha V, Patel FD, Sharma SC et al. Evaluation of intrafraction motion of the organs at risk in image-based brachytherapy of cervical cancer. Brachytherapy 2014; 13: 562-567.

7. Nomden CN, de Leeuw AA, Roesink JM et al. Intra-fraction uncertainties of MRI guided brachytherapy in patients with cervical cancer. Radiother Oncol 2014; 112: 217-220.

8. Pötter R, Kirisits C, Fidarova EF et al. Present status and future of high-precision image guided adaptive brachytherapy for cervix carcinoma. Acta Oncol 2008; 47: 1325-1336.

9. de Andrade Carvalho A, Mendez LC, Stuart SR et al. Implementation of image-guided brachytherapy (IGBT) for patients with uterine cervix cancer: a tumor volume kinetics approach. J Contemp Brachytherapy 2016; 8: 301-307.

10. Pinter C, Lasso A, Wang A et al. SlicerRT: radiation therapy research toolkit for 3D Slicer. Med Phys 2012; 39: 6332-6338.

11. CERR (2017). A computational enviroment for radiotheraoy reaserch. Available at: http:/ / www.cerr.info/about.php

12. Siavashpour Z, Aghamiri MR, Jaberi R et al. Evaluating the utility of "3D Slicer" as a fast and independent tool to assess intrafractional organ dose variations in gynecological brachytherapy. Brachytherapy 2016; 15: 514-523.

13. Patel JL, Goyal RK. Applications of artificial neural networks in medical science. Curr Clin Pharmacol 2007; 2: 217-226.

14. Stergiou C, Siganos D. Neural Networks (2017): Imperial College of London, Computer Department. Available at: http://www.doc.ic.ac.uk/ nd/surprise_96/journal/vol4/ cs11/report.html\#Contents

15. Miller S, Bews J, Kinsner W. Brachytherapy cancer treatment optimization using simulated annealing and artificial neural networks Electrical and Computer Engineering. IEEE, Canada $2001 ; 649-654$.

16. Pecorelli S. Revised FIGO staging for carcinoma of the vulva, cervix, and endometrium. Int J Gynaecol Obstet 2009; 105: 103-104.

17. Petric P, Kirisits C. Potential role of TRAns Cervical Endosonography (TRACE) in brachytherapy of cervical cancer: proof of concept. J Contemp Brachytherapy 2016; 8: 215-220.

18. Kirisits C. The EQD2 concept for practical reporting of cervix brachytherapy. 2014. Available at: www.aoic.net/elekta/ elk1402archive/D201Kirisits.pdf

19. Wyckoff HO, Allisy A, Caswell RS et al. ICRU Report 38: Dose and Volume Specification for Reporting Intracavitary Therapy in Gynecology. International Commission on Radiation Units and Measurements, Maryland 1985.

20. Tanderup K, Nesvacil N, Pötter R et al. Uncertainties in image guided adaptive cervix cancer brachytherapy: impact on planning and prescription. Radiother Oncol 2013; 107: 1-5.

21. Diessner J, Wischnewsky M, Stüber T et al. Evaluation of clinical parameters influencing the development of bone metastasis in breast cancer. BMC Cancer 2016; 16: 307.

22. Rojas R. Neural Networks - A Systematic Introduction. Springer-Verlag, Berlin, New-York 1996. 
23. Knowles JD, Corne DW. Chapter 13 Evolving Neural Networks for Cancer Radiotherapy. In: Hall/CRC C (ed.). School of Computer Science, Cybernetics and Electronic Engineering University of Reading, Whiteknights 2001. Available at: http://ebrary.free.fr/Genetic\%20Algorithms $\% 20$ Handbook/The_Practical_Handbook_of_GA,_v1_Applications,2001/C2409_PDF_C13.pdf.

24. Kurban T, Beşdok E. A Comparison of RBF Neural Network Training Algorithms for Inertial Sensor Based Terrain Classification. Sensors (Basel) 2009; 9: 6312-6329.

25. Heath G, 2016. Available at: https://www.mathworks.com/ matlabcentral/newsreader/view_thread/151286

26. Xie T, Yu H, Wilamowski B. Comparison between Traditional Neural Networks and Radial Basis Function Networks. 2011. Available at: https://pdfs.semanticscholar. org/6d83/51065376ff75086bfd7664eeeae6ef949993.pdf

27. Smith L. 2002. A tutorial on Principal Components Analysis. http://www.cs.otago.ac.nz/cosc453/student_tutorials/ principal_components.pdf

28. Rivard MJ, Coursey BM, deWerd LA et al. Update of AAPM Task Group No. 43 Report: A revised AAPM protocol for brachytherapy dose calculations. Med Phys 2004; 31: 633-674.

29. Analyze Neural Network Performance After Training (1994-2016). The MathWorks, Inc. Available at: https:// www.mathworks.com/help/nnet/ug/analyze-neural-network-performance-after-training.html

30. Kirisits C, Rivard MJ, Baltas D et al. Review of clinical brachytherapy uncertainties: analysis guidelines of GEC-ESTRO and the AAPM. Radiother Oncol 2014; 110: 199-212.

31. Siavashpour Z, Aghamiri MR, Jaberi R et al. Optimum organ volume ranges for organs at risk dose in cervical cancer intracavitary brachytherapy. I Contemp Brachytherapy 2016; 8: 135-142.

32. Orcutt KP, Libby B, Handsfield LL et al. CT-on-rails-guided HDR brachytherapy: single-room, rapid-workflow treatment delivery with integrated image guidance. Future Oncol 2014; 10: 569-575.

33. Leeuw AD. 2016. Image guided brachytherapy: HDR treatments in the MR room. Department of Radiation Oncology, University Medical Center Utrecht, The Netherland. Available at: http://amos3.aapm.org/abstracts/pdf/90-25511-333462108360.pdf.

34. Nesvacil N, Tanderup K, Hellebust TP et al. A multicentre comparison of the dosimetric impact of inter- and intra-fractional anatomical variations in fractionated cervix cancer brachytherapy. Radiother Oncol 2013; 107: 20-25.

35. Purohit A, Asri V, Narula N. Artificial neural networks. Int J Innov Res Technol 2014; 1: 266-270.

36. Willoughby TR, Starkschall G, Janjan NA et al. Evaluation and scoring of radiotherapy treatment plans using an artificial neural network. Int J Radiat Oncol Biol Phys 1996; 34: 923-930.

37. Hosseini-Ashrafi ME, Bagherebadian H, Yahaqi E. Pre-optimization of radiotherapy treatment planning: an artificial neural network classification aided technique. Phys Med Biol 1999; 44: 1513-1528.

38. Orr MJL. 1996. Introduction to Radial Basis Function Networks. University of Edinburgh. Available at: http://www. cc.gatech.edu/ isbell/tutorials/rbf-intro.pdf 